\title{
Ocorrência de genes tad associados à formação de biofilme em isolados de Pasteurella multocida de pulmões de suínos com pneumonia ${ }^{1}$
}

INDEX TERMS: Swine, Pasteurella multocida, gene tad, pneumonia.

\begin{abstract}
RESUMO.- Os atuais sistemas de criação intensiva de suínos aumentam a pressão de seleção microbiana propiciando a disseminação de doenças respiratórias. A bactéria Pasteurella multocida é associada a diversas patologias respiratórias em animais submetidos a esse tipo de criação, causando grandes perdas econômicas. A formação de biofilme foi descrita in vitro em $P$. multocida e fatores analisados indicaram a facilitação na colonização dos tecidos,

\footnotetext{
${ }^{1}$ Recebido em 28 de fevereiro de 2014.

Aceito para publicação em 7 de julho de 2014.

${ }^{2}$ Laboratório de Microbiologia e Biologia Molecular Veterinária, Departamento de Clínica Médica Veterinária do Hospital Veterinário, Universidade Federal do Mato Grosso (UFMT), Av. Fernando Corrêa da Costa 2367, Bairro Boa Esperança, Cuiabá, MT 78060-900, Brasil. *Autor para correspondência: valdutra@ufmt.br

${ }^{3}$ Embrapa Suínos e Aves, Cx. Postal 21, Concordia, SC 89700-000, Brasil.
}

aumentando a resistência às defesas do hospedeiro e aos antibióticos. Os objetivos deste trabalho foram analisar a ocorrência de P. multocida em pneumonias de suínos e na microbiota de pulmões sem lesão e a ocorrência dos genes do lócus tad nestes isolados. Foram analisados 70 isolados de P. multocida de pulmões, sendo sessenta e sete com lesão e três sem lesão. Isolados do sorotipo A ocorreram principalmente em pulmões com lesões $(85,71 \%)$, enquanto em pulmões sem lesão observou-se somente o sorotipo D. Os genes tadA, tadB, tadC, tadD, tadE tadF e tadG estavam presentes em $89,55 \%$ dos isolados de pulmões com lesões. Os genes $\operatorname{tadA}, \operatorname{tad} B$ e $\operatorname{tad} C$ estavam presentes em todos os isolados de pulmões sem lesão, porém os genes tadD, tadE, tadF e tadG estavam presentes em $0 \%, 33,3 \%$, $33,3 \%$ e $66,6 \%$, dos isolados sem lesão, respectivamente. Neste trabalho observou-se a associação da ocorrência dos 
genes tadD, tadE e tadF em isolados de P. multocida e a presença de lesões em pulmões.

TERMOS DE INDEXAÇÃO: Suínos, Pasteurella multocida, genes tad, pneumonia.

\section{INTRODUÇÃO}

Pasteurella multocida (P. multocida) é um bacilo Gram-negativo capaz de causar doenças em várias espécies, levando a perdas econômicas, especialmente associadas às indústrias de processamento de produtos de origem animal, no mundo todo (Borowski 2001, Ribeiro et al. 2012). Esta bactéria é transmitida por pequenas quantidades de partículas suspensas no ar e pelo contato direto com animais doentes, sendo capaz de causar patologias como a cólera aviário, septicemia e pneumonia em bovinos, rinite atrófica progressiva, pleurisia e pneumonia em suínos (Boyce et al. 2010). Uma vez que faz parte da microbiota da mucosa oral de cães e gatos, casos de pasteurelose em humanos são geralmente associadas ao contato com esses animais, através de mordeduras e/ou arranhaduras (Collins et al. 2012, Nagata et al. 2013, Nguefack et al. 2014).

São descritos cinco sorotipos: $A, B, D, E$ e $F$ de acordo com os antígenos capsulares (Townsed et al. 2001), sendo geralmente associado, mas não restrito, a um hospedeiro específico (Arumugam et al. 2011). Diferentes técnicas de genotipificação têm sido utilizadas para classificar este microrganismo, como o RAPD (Lee et al. 2011) e MLST (Silva et al. 2012, Bisgaard et al. 2013). Dada a importância econômica desta bactéria, principalmente em sistemas de produção de suínos, seus fatores de virulência têm sido amplamente estudados.

A toxina PMT está geralmente associada a amostras toxigênicas e outras bactérias, como a Bordetella bronchiseptica (B. bronchiseptica), responsáveis por causar rinite atrófica nos suínos (Bäckström et al. 1988, Ahn et al. 2008). São descritos também, os genes exbB/tonBe $h g b A$ envolvidos no sequestro de ferro, os genes codificadores da neuraminidase (nanB e nanH), fímbria do tipo IV e proteínas de membrana externa (OMP e Oma87) (Ruffolo \& Alder 1996, Bethe et al. 2009). Além de dez genes associados à utilização de L-fucose, responsável pelo metabolismo de carboidratos, fundamentais aos processos bacterianos para aquisição de energia em ambiente de baixa disponibilidade de nutrientes, como o trato respiratório (Johnson et al. 2013).

Os genes do lócus tad são essenciais para formação de biofilme em vários gêneros, como Aggregatibacter, Haemophilus, Pasteurella, Pseudomonas, Yersinia e Caulobacter (Tomich et al. 2007). Com seu lócus gênico composto por flp1, flp2, tadV, rcpC, rcpA, rcpB, tadZ, tadA, tadB, tadC, tadD, tadE, tadF e tadG ou apenas flp-rcp-tad, têm sido implicado na patogênese de diversos gêneros bacterianos (Tomich et al. 2007). Schreiner et al. (2003) relatam que a mutação nos genes flp-1 e tadA causaram a perda na virulência de amostras patogênicas oriundas de humanos, da região da cavidade oral. Harper et al. (2006) e Tang et al. (2009) citam o tadD como um fator de patogenicidade crucial à aderências em substratos (tecidos ou materiais) e formação de biofilme em P. multocida.
O objetivo deste trabalho foi analisar a ocorrência de P. multocida em pneumonias de suínos e da microbiota de pulmões sem lesão e a ocorrência dos genes do lócus tad nestes isolados.

\section{MATERIAL E MÉTODOS}

Amostras foram coletados de 100 pulmões com lesões macroscópicas de pneumonia (consolidação pulmonar, deposição de fibrina, pleurisia e/ou aderência) e 100 pulmões sem lesões macroscópicas em frigoríficos de Inspeção Federal entre 2010 e 2012. Os pulmões foram submetidos a exames microbiológicos para isolamento de Pasteurella multocida e estes foram confirmados bioquimicamente pelos testes da catalase, oxidade, urease, Triple Sugar Iron (TSI) e os açúcares: glicose, sacarose, lactose, maltose e manitol (Quinn et al. 1994).

Extração de DNA e PCR específico para $P$. multocida e sorotipos dos isolados. Para confirmação do gênero, espécie e sorotipos capsulares através do PCR foram utilizados os seguintes genes: kmt1 (P. multocida), hyaD-hyaC (sorotipo A), bcbD (sorotipo B), $d c b F$ (sorotipo D), ecbJ (sorotipo E) e $f c b D$ (sorotipo F). O DNA foi extraído, seguindo o protocolo de fenol/clorofórmio (Sambrook \& Russel 2004). A PCR específica para o gene kmt1 e para os sorotipos foram realizadas a partir do proposto por Townsed et al. (2001) (volume final de $20 \mu \mathrm{l}$, 20ng de DNA, 2.4mM de $\mathrm{MgCl}_{2}, 1 \mathrm{x}$ de Taq Buffer com 50mM de KCl, 1,0 $\mu \mathrm{M}$ de cada oligonucleotídeo, $0,2 \mathrm{mM}$ de dNTPs e $1 \mathrm{U}$ Taq DNA polimerase). As amplificações para todos os genes foram realizadas em termociclador (BIO-RAD MyCyclerThermalCycler), com desnaturação inicial por 5 minutos a $95^{\circ} \mathrm{C}$, seguido de 30 ciclos de 30 segundos para desnaturação a $95^{\circ} \mathrm{C}, 30$ segundos de anelamento a $54^{\circ} \mathrm{C}$ e 60 segundos de extensão a $72^{\circ} \mathrm{C}$, seguidos por um ciclo de extensão final por 7 minutos a $72^{\circ} \mathrm{C}$. Como controle positivo foi utilizado o isolado M135/10 de P. multocida, confirmado por sequenciamento do gene kmt1 e genotipado por MLST (Multilocus Sequence Typing) com as sequências depositadas no PubMLST (http://pubmlst.org/pmultocida)(Silva et al. 2012), e como controle negativo, água ultrapura. Os produtos de amplificação foram analisados em eletroforese em gel agarose 2,0\%, corados com Gel $\operatorname{Red}^{m}\left(\right.$ Biotium $\left.^{\circledR}\right)$, a $10 \mathrm{~V}$ por cm, e observados em ChemiDoc ${ }^{\text {TM }}$ XRS utilizando o software Image$\mathrm{Lab}^{\mathrm{TM}}$. Como marcador de massa molecular utilizou-se o padrão 100 pb DNA Ladder ${ }^{\mathrm{m}}$ (Fermentas $\left.{ }^{\circledR}\right)$. Os oligonucleotídeos utilizados e tamanho dos amplicons encontram-se no Quadro 1.

PCR para os genes tad. Para análise da presença dos genes tad, foram desenhados sete pares de oligonucleotídeos, sendo o tadA, tadB, tadC, tadD, tadE, tadF e tadG (Quadro 3). Todos os oligonucleotídeos foram desenhados com base na sequência da amostra de P. multocida PM36950 (GenBank CP003022) utili-

Quadro1. Identificação, sequências e altura de amplificação dos sorotipos de Pasteurella multocida

\begin{tabular}{cccc}
\hline Sorogrupo & Gene & Sequência 5' 3' & $\begin{array}{c}\text { Tamanho de } \\
\text { amplificação }\end{array}$ \\
\hline Todos & kmt1 & $\begin{array}{l}\text { ATCCGCTATTTACCCAGTGG } \\
\text { GCTGTAAACGAACTCGCCAC }\end{array}$ & 460 \\
A & hyaD-hyaC & $\begin{array}{l}\text { TGCCAAAATCGCAGTCAG } \\
\text { TTGCCATCATTGTCAGTG }\end{array}$ & 1.044 \\
B & bcbD & $\begin{array}{l}\text { CATTTATCCAAGCTCCACC } \\
\text { GCCCGAGAGTTCAATCC }\end{array}$ & 760 \\
D & dcbF & $\begin{array}{l}\text { TTACAAAAGAAAGACTAGGAGCCC } \\
\text { CATCTACCCACTCAACCATATCAG }\end{array}$ & 657 \\
E & ecbJ & $\begin{array}{l}\text { TCCGCAGAAATTATTGACTC } \\
\text { GCTTGCTGCTTGATTTGTC }\end{array}$ & 511 \\
F & fcbD & $\begin{array}{l}\text { AATCGGAGAACGCAGAAATCAG } \\
\text { TTCCGCCGTCAATTACTCTG }\end{array}$ & 851
\end{tabular}


Quadro 3. Sequência de oligonucleotídeos dos genes tad e seus respectivos tamanhos de amplicons

\begin{tabular}{|c|c|c|}
\hline TAD & Sequencia 5' 3' & $\begin{array}{l}\text { Tamanho de } \\
\text { amplificação }\end{array}$ \\
\hline \multirow[t]{2}{*}{ TAD A1 } & Foward: GGTCGCATCACGAGGACTAT & \\
\hline & Reverse: AGGCGAAATTACGATGCAAG & $172 \mathrm{pb}$ \\
\hline \multirow[t]{2}{*}{ TAD B } & Forward: TTCGCCTAATTGTCCCGTTA & \\
\hline & Reverse: TGGAAGTTAGGGCAATACCG & $150 \mathrm{pb}$ \\
\hline \multirow[t]{2}{*}{ TAD C } & Forward: GTGACCAAAAGCTTCACACG & \\
\hline & Reverse: GGGATTGCAGTTTTGCTGAT & $221 \mathrm{pb}$ \\
\hline \multirow[t]{2}{*}{ TAD D } & Foward: TCGCAACCGCTAATCGACAA & \\
\hline & Reverse: GCGGCTTTAAAACAAGTGGC & $1274 \mathrm{pb}$ \\
\hline \multirow[t]{2}{*}{ TAD E } & Foward: TGGATTCGTCCCAAGAGAAC & \\
\hline & Reverse: ATCTCTCCTACGGGGAGTCG & $195 \mathrm{pb}$ \\
\hline \multirow[t]{2}{*}{ TAD F } & Foward: GATCTAATCAGCCCCGTTGA & \\
\hline & Reverse: TATCATTGGCGATTGTACGC & $183 \mathrm{pb}$ \\
\hline \multirow[t]{2}{*}{ TAD G } & Foward: AACTTGCCCAATTGTTCTCG & \\
\hline & Reverse: CCTTCTGGTTGGACTTCTGC & $224 \mathrm{pb}$ \\
\hline
\end{tabular}

zando a ferramenta primer-BLAST ${ }^{\text {'m }}$ (disponível em: http://www. ncbi.nlm.nih.gov/tools/primer-blast/).

A amplificação dos genes através da PCR foi realizada em um volume final de $20 \mu \mathrm{l}$, contendo $30 \mathrm{ng}$ de DNA, $20 \mathrm{pmol}$ de cada oligonucleotídeo, 2,5mM de $\mathrm{MgCl}_{2}$, 1,5U de Taq DNA polimerase (Invitrogen ${ }^{\circledR}$ ), $1 \mathrm{x}$ de taq buffer com $50 \mathrm{mM}$ de $\mathrm{KCl}, 0,2 \mathrm{mM}$ de cada dNTP e água ultra pura q.s.p. Os produtos de amplificação foram analisados em eletroforese em gel agarose 2,0\%, corados com $\mathrm{Gel}$ $\operatorname{Red}^{\mathrm{Tm}}$ (Biotium ${ }^{\circledR}$ ), a $10 \mathrm{~V}$ por cm e observados em ChemiDoc ${ }^{\mathrm{TM}} \mathrm{XRS}$ utilizando o software Image Lab ${ }^{\mathrm{TM}}$.

Análise estatística. Foi utilizada a análise estatística de regressão logística multivariada para verificar associação entre as variáveis sorotipo, presença de lesão pulmonar e presença dos genes do lócus tad nos isolados de P. multocida. As variáveis com interações significativas $(p<0,05)$ foram posteriormente analisadas pelo teste exato de Fisher. Todas as análises foram realizadas pelo programa R versão 2.14.2 (R Foundation for Statistical Computing, Vienna, Austria).

\section{RESULTADOS}

Foram obtidos 70 isolados, identificados pelos testes bioquímicos e amplificação do gene kmt1 específico para Pasteurella multocida (Quadro 2). Destes, 67 (67/70) isolados eram de pulmões que apresentavam alterações macroscópicas e apenas três isolados $(3 / 70)$ foram de pulmões sem lesões. Na tipificação capsular, 85,71\% (60/70) das amostras de $P$. multocida foram positivas para o sorotipo capsular A e $14,29 \%$ para o sorotipo D (10/70).

$\mathrm{Na}$ análise da ocorrência dos genes do lócus tad observou-se que todos os isolados foram positivos para os genes $\operatorname{tad} A, \operatorname{tad} B$ e $\operatorname{tad} C$. Os genes $t a d D, t a d E$, $t a d F$ e $\operatorname{tad} G$, entretanto, apresentaram variações na ocorrência com valores de $85,71 \%, 97,14 \%, 97,14 \%$, e $98,57 \%$, respectivamente.

A análise estatística através da regressão logística multivariada mostrou associação entre sorotipo, presença de lesão e ocorrência dos genes tad em isolados de P. multocida. Ocorreu também associação entre a presença de lesão e o isolados do sorotipo capsular A $(\mathrm{p}=0,02)$, sendo as amostras isoladas de pulmões sem lesões somente do sorotipo D.

A frequência de ocorrência dos genes do lócus tad também apresentou associação com o sorotipo capsular. 0 gene tadD esteve ausente nos dez isolados do sorotipo D. A ocor- rência dos genes tadE, tadF e tadG também foi menor em isolados do sorotipo $\mathrm{D}$, sendo estatisticamente significativa a ausência dos genes $\operatorname{tad} E(\mathrm{p}=0,018)$ e $\operatorname{tad} F(\mathrm{p}=0,018)$.

Com relação à ocorrência dos genes do lócus tad em isolados de pulmões com e sem lesões, observou-se que os genes tad estavam presentes em $100 \%$ dos isolados de $P$. multocida de pulmões com lesões, exceto o tadD (Figura 1). Já nos três isolados provenientes de pulmões sem lesão, os genes tadA, tadB, tadC estavam presentes em 100\% (3/3) das amostras (Fig.1). Os genes tadE, tadF e tadG estavam presentes em 33,3\% (1/3), 33,3\% (1/3) e 66,6\% (2/3), respectivamente, nos isolados. No caso do gene tadD, todos os isolados (0/3) foram negativos (Fig.1).

Pela análise do teste de Fisher foi observada a associação existente entre a presença de lesão e a ocorrência de genes $\operatorname{tad} D(\mathrm{p}=0,002), \operatorname{tad} E(\mathrm{p}=0,001)$ e $\operatorname{tadF}(\mathrm{p}=0,001)$ nos isolados analisados.

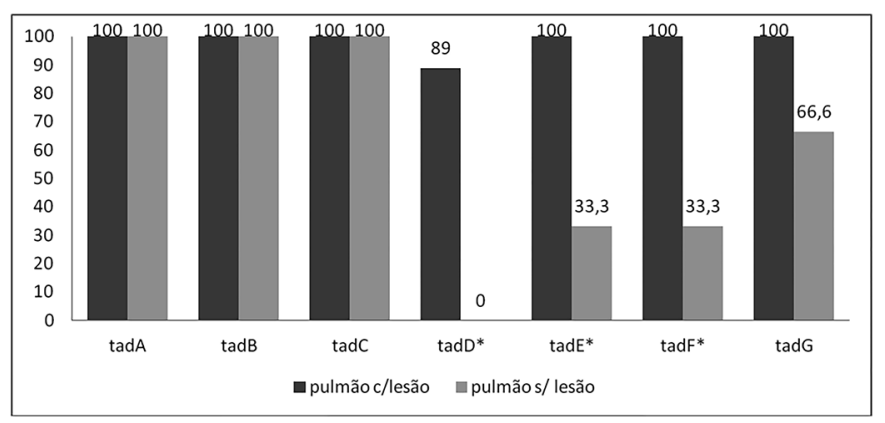

Fig.1. Percentual de ocorrência de genes tad em isolados de P. multocida de pulmões com e sem lesões macroscópicas. Os genes marcados com * apresentam diferença na ocorrência nos pulmões com e sem lesões $(\mathrm{p}<0,05)$.

\section{DISCUSSÃO}

Pasteurelose é uma das doenças mais comuns em suínos de crescimento e terminação em todo o mundo. Amostras toxigênicas, capazes de desencadear a rinite atrófica progressiva (RAP), são associadas principalmente aos sorotipos $D$ (Amigot et al. 1998). Os isolados de pneumonia geralmente são não toxigênicos e podem apresentar os tipos capsulares $A$ e $D$, sendo o sorotipo A o mais prevalente (Ewers et al. 2006, Bethe et al. 2009). Destacam-se os maiores índices encontrados em pulmões com lesões de pneumonia associados ao sorotipo A, em vários países como o Reino Unido $(82,2 \%)$, Alemanha $(53,4 \%)$ e Coréia do Sul $(56,6 \%)$ (Davies et al. 2003, Ahn et al. 2008, Bethe et al. 2009). Tang et al. (2009) relataram a presença do sorotipo capsular A em apenas $39,5 \%$ das amostras de um total de 233 isolados de P. multocida obtidos de suínos com doença clínica respiratória, na China. Ainda neste mesmo estudo, o sorotipo D foi identificado em 54,9\% dos isolados. Provavelmente esses valores se devem ao fato de neste estudo terem sido avaliados isolados de vários locais do trato respiratório, sem descrever a origem tecidual da amostra, ao contrário dos demais estudos que analisaram apenas os de origem pulmonar. No Brasil os índices do sorotipo A encontrados variam entre 89,4\% (Moreno et al. 2003) a 95,5\% (Borowski et al. 2002), índices similares encontrados em nosso estudo $(85,71 \%)$. 
Quadro 2. Isolados de Pasteurella multocida com resultado de sorotipo capsular, ocorrência de lesão e presença dos genes tad

\begin{tabular}{|c|c|c|c|c|c|c|c|c|c|}
\hline Isolado S & Sorotipo & Lesão & $\operatorname{tad} A$ & $\operatorname{tad} B$ & $\operatorname{tad} C$ & $\operatorname{tad} D$ & $\operatorname{tad} E$ & $\operatorname{tad} F$ & $\operatorname{tad} G$ \\
\hline M305/09 & $\mathrm{A}$ & Presente & + & + & + & + & + & + & + \\
\hline M135/10 & A & Presente & + & + & + & + & + & + & + \\
\hline M142/10 & A & Presente & + & + & + & + & + & + & + \\
\hline M150/10 & A & Presente & + & + & + & + & + & + & + \\
\hline M152/10 & A & Presente & + & + & + & + & + & + & + \\
\hline M153/10 & A & Presente & + & + & + & + & + & + & + \\
\hline M154/10 & A & Presente & + & + & + & + & + & + & + \\
\hline M155/10 & A & Presente & + & + & + & + & + & + & + \\
\hline M158/10 & A & Presente & + & + & + & + & + & + & + \\
\hline M159/10 & A & Presente & + & + & + & + & + & + & + \\
\hline M160/10 & A & Presente & + & + & + & + & + & + & + \\
\hline M161/10 & A & Presente & + & + & + & + & + & + & + \\
\hline M162/10 & A & Presente & + & + & + & + & + & + & + \\
\hline M163/10 & A & Presente & + & + & + & + & + & + & + \\
\hline M164/10 & A & Presente & + & + & + & + & + & + & + \\
\hline M165/10 & A & Presente & + & + & + & + & + & + & + \\
\hline M166/10 & A & Presente & + & + & + & + & + & + & + \\
\hline M167/10 & A & Presente & + & + & + & + & + & + & + \\
\hline M171/10 & A & Presente & + & + & + & + & + & + & + \\
\hline M173/10 & A & Presente & + & + & + & + & + & + & + \\
\hline M174/10 & A & Presente & + & + & + & + & + & + & + \\
\hline M175/10 & A & Presente & + & + & + & + & + & + & + \\
\hline RS/G09/02 & D & Presente & + & + & + & - & + & + & + \\
\hline $\mathrm{MT} / \mathrm{G} 04 / 03$ & $\mathrm{D}$ & Presente & + & + & + & - & + & + & + \\
\hline MG/F01/01 & $\mathrm{D}$ & Presente & + & + & + & - & + & + & + \\
\hline MG/F01/02 & $\mathrm{D}$ & Presente & + & + & + & - & + & + & + \\
\hline MG/F01/03 & $\mathrm{D}$ & Presente & + & + & + & - & + & + & + \\
\hline MG/F08/01 & $\mathrm{D}$ & Presente & + & + & + & - & + & + & + \\
\hline MS/F08/01 & $\mathrm{D}$ & Presente & + & + & + & - & + & + & + \\
\hline MTF04/01 & A & Presente & + & + & + & + & + & + & + \\
\hline MTF02/02 & A & Presente & + & + & + & + & + & + & + \\
\hline MTF03/02 & A & Presente & + & + & + & + & + & + & + \\
\hline MTF06/02 & A & Presente & + & + & + & + & + & + & + \\
\hline MTG05/01 & A & Presente & + & + & + & + & + & + & + \\
\hline MTF01/01 & A & Presente & + & + & + & + & + & + & + \\
\hline MTF03/01 & A & Presente & + & + & + & + & + & + & + \\
\hline MTF01/02 & A & Presente & + & + & + & + & + & + & + \\
\hline MTF02/01 & A & Presente & + & + & + & + & + & + & + \\
\hline MTF03/02 & A & Presente & + & + & + & + & + & + & + \\
\hline MTG04/02 & A & Presente & + & + & + & + & + & + & + \\
\hline $1 \mathrm{~B}$ & A & Presente & + & + & + & + & + & + & + \\
\hline $2 \mathrm{~B}$ & A & Presente & + & + & + & + & + & + & + \\
\hline $3 B$ & A & Presente & + & + & + & + & + & + & + \\
\hline $6 \mathrm{~B}$ & A & Presente & + & + & + & + & + & + & + \\
\hline $11 \mathrm{~B}$ & A & Presente & + & + & + & + & + & + & + \\
\hline $12 \mathrm{~B}$ & A & Presente & + & + & + & + & + & + & + \\
\hline $13 \mathrm{~B}$ & A & Presente & + & + & + & + & + & + & + \\
\hline 14B & A & Presente & + & + & + & + & + & + & + \\
\hline 15B & A & Presente & + & + & + & + & + & + & + \\
\hline $16 \mathrm{~A}$ & $\mathrm{D}$ & Ausente & + & + & + & - & + & + & + \\
\hline $16 \mathrm{~B}$ & A & Presente & + & + & + & + & + & + & + \\
\hline $17 \mathrm{~A}$ & $\mathrm{D}$ & Ausente & + & + & + & - & - & - & - \\
\hline 17B & A & Presente & + & + & + & + & + & + & + \\
\hline $18 \mathrm{~A}$ & $\mathrm{D}$ & Ausente & + & + & + & - & - & - & + \\
\hline $20 B$ & A & Presente & + & + & + & + & + & + & + \\
\hline $25 B$ & A & Presente & + & + & + & + & + & + & + \\
\hline $26 B$ & A & Presente & + & + & + & + & + & + & + \\
\hline $27 B$ & A & Presente & + & + & + & + & + & + & + \\
\hline $28 B$ & A & Presente & + & + & + & + & + & + & + \\
\hline $31 B$ & A & Presente & + & + & + & + & + & + & + \\
\hline $32 B$ & A & Presente & + & + & + & + & + & + & + \\
\hline $33 B$ & A & Presente & + & + & + & + & + & + & + \\
\hline $35 B$ & A & Presente & + & + & + & + & + & + & + \\
\hline $41 B$ & A & Presente & + & + & + & + & + & + & + \\
\hline $43 B$ & A & Presente & + & + & + & + & + & + & + \\
\hline $44 B$ & A & Presente & + & + & + & + & + & + & + \\
\hline $45 B$ & A & Presente & + & + & + & + & + & + & + \\
\hline $46 B$ & A & Presente & + & + & + & + & + & + & + \\
\hline $47 B$ & A & Presente & + & + & + & + & + & + & + \\
\hline $48 B$ & A & Presente & + & + & + & + & + & + & + \\
\hline
\end{tabular}


A capacidade de formar biofilme aumenta a patogenicidade bacteriana, pois impede a ação de antimicrobianos e do sistema monocítico fagocitário (Donlan \& Conserton 2002). 0 lócus tad pertence ao grupo das adesinas e é composto por 14 genes, sendo eles o flp-1-flp-2-tadV-rcpCABtadZABCDEFG, sendo responsável pela aderência e formação de biofilme em várias espécies patogênicas, como o Actinobacillus actinomicetecomytans, Yersinia pestis, Vibrio cholerae, Mycobacterium tuberculosis, Pseudomonas aeruginosa e Bordetella pertussis (Kachlany et al. 2000, Tomich et al. 2007, Bernard et al. 2009, Schilling et al. 2010).

Ao menos 12 genes do lócus tad são requeridos para a formação de todos os fenótipos relacionados à aderência, incluindo a produção do Flp pili, morfologia rugosa das colônias, auto agregação e formação de biofilme (Tomich et al. 2007). Harper et al. (2006) citam que as fímbrias e adesinas são fundamentais para a aderência em células do hospedeiro e que o tadD atua codificando um aparato de secreção requerido para a formação do Flp pili. Fuller et al. (2000) demonstraram que mutações no gene tadD de $P$. multocida tornaram a virulência da bactéria atenuada. Isto poderia explicar nossos resultados, onde o gene tadD foi encontrado na maioria das amostras oriundas de pulmões com lesão 89,55\% (60/67) e estava ausente nas amostras sem lesão (0/3), apesar de um pequeno numero dessas amostras, devido à baixa prevalência de P. multocida na microbiota dos pulmões sem lesão.

Tang et al. (2009) analisando genes de virulência em 233 isolados de $P$. multocida de lesões pulmonares e rinite atrófica obtiveram 101 (43,3\%; 101/233) dos isolados positivos para o gene tadD. Neste estudo, ficou evidenciada uma correlação estatística entre o sorotipo capsular A e o gene tadD.

Alguns autores demonstraram que mutações nos genes tadE e tadF em A. actinomycetecomitans ocasionam uma diminuição na produção de pili e incapacidade de adesão em superfície (Kachlany et al. 2000, Wang et al. 2003, Tomich et al. 2006). Nos isolados do nosso estudo apenas dois não apresentaram amplificação para esses genes (17A e 18A), sendo ambos de pulmões sem lesão, indicando que possa ter ocorrido uma baixa capacidade de fixação no epitélio pulmonar por parte destes.

Em P. multocida a ocorrência de genes do lócus tad poderia facilitar a adesão da bactéria nas células hospedeiras e ser um fator de virulência importante na ocorrência de pneumonia em suínos. Essas novas variantes genéticas provavelmente originaram-se da necessidade de adaptação da bacteria ao hospedeiro, através de trocas horizontais de materiais genéticos, conhecido como "ilha genômica” (Hacker \& Kaper 2000). Análises das $\gamma$-proteobactérias, incluindo a família Pasteurellaceae indicam que a transferência horizontal dessas ilhas pode ocorrer entre famílias próximas e estudos mostram que existe uma grande dispersão entre os procariotos (Planet et al. 2003).

\section{CONCLUSÃO}

Os genes tadD, tadE e tadF importantes na adesão e formação de biofilme em P. multocida estão associados a presença de lesões pulmonares em suínos.
Agradecimentos.- À Coordenação de Aperfeiçoamento de Pessoal de Nível Superior (CAPES), pela concessão da bolsa de mestrado e à Fundação de Amparo a Pesquisa do Estado de Mato Grosso (Fapemat) pelo financiamento do projeto.

\section{REFERÊNCIAS}

Ahn K.K., Lee Y.H., Ha Y., Kim D., Chae S., Kim C.H., Lee J.H., Kim S.H. \& Chae C. 2008. Detection by in-situ hybridization of Pasteurella multocida toxin (toxA) gene in the lungs of naturally infected pigs. J. Comp. Pathol. 139(1):51-53.

Amigot J.A., Torremorell M. \& Pijoan C. 1998. Evaluation of techniques for the detection of toxigenic Pasteurella multocida strains from pigs. J. Vet. Diagn. Invest. 10:169-173.

Arumugam N.D., Ajam N., Blackall P.J., Asiah N.M., Ramlan M., Maria J., Yuslan S. \& Thong K.L. 2011. Capsular serotyping of Pasteurella multocida from various animal hosts - a comparison of phenotypic and genotypic methods. Trop. Biomed. 28(1):55-63.

Bäckström L.R., Brim T.A. \& Collins M.T. 1988. Development of turbinate lesions and nasal colonization of Bordetella bronchiseptica and Pasteurella multocida during long term exposure of healthy pigs affected by atrophic rhinitis. Can. J. Vet. Res. 52:23-29.

Bernard C.S., Bordi C., Termine E., Filloux A. \& Bentzmann S. 2009. Organization and PprB-dependent control of the Pseudomonas aeruginosa tad locus, involved in Flp pilus biology. J. Bacteriol. 191(6):1961-1973.

Bethe A., Wieler L.H., Selbitz H.J. \& Ewers C. 2009. Genetic diversity of porcine Pasteurella multocida strains from the respiratory tract of healthy and diseased swine. Vet. Microbiol. 139(1/2):97-105.

Bisgaard M., Petersen A. \& Christensen H. 2013. Multilocus sequence analysis of Pasteurella multocida demonstrates a type species underdevelopment. Microbiol. 159:580-590.

Borowski S.M. 2001. Pasteurelose pulmonar: uma atualização. Anais X Congresso da Abraves, Porto Alegre, RS, p. 8. (Resumo)

Borowski S.M., Ikuta N., Lunge V., Fonseca A., Marques E. \& Cardoso M. 2002. Caracterização antigênica e fenotípica de cepas de Pasteurella multocida isoladas de pulmões de suínos com pneumonia e/ou pleurite. Pesq. Vet. Bras. 22(3):97-103.

Boyce J.D., Harper M., Wilkie I.W. \& Adler B. 2010. Pasteurella, p.325-340. In: Prescott J.F. (Ed.), Pathogenesis of Bacterial Infections in Animals. $4^{\text {th }}$ ed. Blackwell Publishing, Ames.

Collins C., Flanagan B. \& Henning J.S. 2012. An atypical presentation of a Pasteurella multocida infection following a cat bite: a case report. Cutis 89(6):269-72.

Davies R.L., MacCorquodale R., Baillie S. \& Caffrey B. 2003. Characterization and comparison of Pasteurella multocida strains associated with porcine pneumonia and atrophic rhinitis. J. Med. Microbiol. 52:59-67.

Donlan R.M. \& Conserton J.W. 2002. Biofilms: Survival mechanisms of clinically relevant microorganisms. Clin. Microbiol. Rev. 15(2):167-193.

Ewers C., Lübke-Becker A., Bethe A., Kiebling S., Filter M. \& Wieler L.H. 2006. Virulence genotype of Pasteurella multocida strains isolated from different hosts with various disease status. Vet. Microbiol. 114:304-317.

Fuller T.E., Kennedy M.J. \& Lowery D.E. 2000. Identification of Pasteurella multocida virulence genes in a septicemic mouse model using signature-tagged mutagenesis. Microbiol. Pathogens 29:25-38.

Hacker J. \& Kaper J.B. 2000. Pathogenicity islands and the evolution of microbes. Rev. Microbiol. 54:641-79.

Harper M., Boyce J.D. \& Adler B. 2006. Minireview Pasteurella multocida pathogenesis: 125 years after Pasteur FEMS Microbiol. Lett. 265:1-10.

Johnson T., Abrahante J.E., Hunter S.S., Hauglund M., Tatum F.M., Maheswaran S.K. \& Briggs R.E. 2013. Comparative genome analysis of an avirulent and two virulent strains of avian Pasteurella multocida reveals candidate genes involved in fitness and pathogenicity. BMC Microbiol. 14(3):106.

Kachlany S.C., Planet P.J., Bhattacharjee M.K., Kollia E., Desalle R., Fine D.H. \& Figurski D.H. 2000. Nonspecific adherence by Actinobacillus actinomycetemcomitans requires genes widespread in bacteria and archaea. J. Bacteriol. 182(21):6169-6176. 
Lee K.E., Jeoung H.Y., Lee J.Y., Lee M.H., Choi H.W. \& Chang K.S. 2011. Phenotypic characterization and Random Amplified Polymorphic DNA (RAPD)analysis of Pasteurella multocida isolated from Korean pigs. J. Vet. Med. Sci. 74(5):567-573.

Moreno A.M., Baccaro M.R., Ferreira A.J. \& De Castro A.F.P. 2003. Use of single-enzyme amplified fragment length polymorphism for typing Pasteurella multocida subsp. multocida isolates from pigs. J. Clin. Microbiol. 41(4):1743-1746.

Nagata H., Yamada S., Uramaru K., Kiyasu Y. \& Kano N. 2013. Acute cholecystitis with bacteremia caused by Pasteurella multocida. Surg. Infect. 14:1-3.

Nguefack S., Moifo B., Chiabi A., Mah E., Bogne J.B., Fossi M., Fru F., Mbonda E. \& Djientcheu V.P. 2014. Méningite à Pasteurella multocida compliquée d'abcès cérébral. Arch. Pediatr. 3571:1-3.

Planet P.J., Kachlany S.C., Fine D.H., DeSalle R. \& Figurski D.H. 2003. The widespread colonization island of Actinobacillus actinomycetemcomitans. Nat. Gen. 24(2):193-198.

Quinn P.J., Carter M.E., Markey B. \& Carter G.R. 1994. Clinical veterinary microbiology. Wolfe, London.

Ribeiro W.L.C., Pinheiro A.R.A., Evangelista J.N.B. \& Sales R.O. 2012. Rinite atrófica e sua importância na indústria suinícola: uma revisão. Revta Bras. Hig. Sanid. Anim. 6(1):21-35.

Ruffolo C.G. \& Alder B. 1996. Cloning, sequencing, expression, and protective capacity of theoma87 gene encoding the Pasteurella multocida 87-Kilodalton outer membrane antigen. Infect. Immun. 64(8):31613167.

Sambrook J. \& Russel D.W. 2004. Molecular cloning: a laboratory manual. $3^{\text {rd }}$ ed. Cold Spring Harbor Laboratory Press, New York, p.5.65-5.67.
Schilling J., Wagner K., Seekircher S., Greune L., Humberg V., Schmidt M.A. \& Heusipp G. 2010. Transcriptional activation of the tad type IVb pilus operon byPypB in Yersinia enterocolitica. J. Bacteriol. 192(14):38093821.

Schreiner H.C., Sinatra K., Kaplan J.B., Furgang D., Kachlany S.C., Planet P.J., Perez B.A., Figurski D.H. \& Fine D.H. 2003. Tight-adherence genes of Actinobacillus actinomycetemcomitans are required for virulence in a rat model. PNAS 100(12):7295-7300.

Silva G.F.R., Brandão L.N.S., Paula D.A.J., Pescador C.A., Chitarra C.S., Carvalho R.C.T., Nakazato L. \& Dutra V. 2012. Characterization using Multilocus Sequence Typing and virulence fators of Pasteurella multocida from pigs with pneumonia in states of Mato Grosso and Mato Grosso do Sul, Brazil. Vet. Sci. Res. 3(1):55-59.

Tang X., Zhao Z., Hu J., Wu B., Cai X., He Q. \& Chen H. 2009. Isolation, antimicrobial resistance, and virulence genes of Pasteurella multocida strains from swine in China. J. Clin. Microbiol. 47(4):951-958.

Tomich M., Planet P.J. \& Figurski D.H. 2007. The tad locus: postcards from the widespread colonization island. Nat. Rev. 5:363-375.

Tomich M., Fine D.H. \& Figurski D.H. 2006. The tadV protein of Actinobacillus actinomycetemcomitans is anovel aspartic acid prepilin peptidase required for maturation of the Flp1 pilin and $t a d E$ and $t a d F$ pseudopilins. J. Bacteriol. 188(19):6899-6914.

Townsed K., Boyce J.D., Chung J.Y., Frost A.J. \& Alder B. 2001. Genetic organization of Pasteurella multocida cap loci and development of a multiplex capsular PCR typing system. Clin. Microbiol. 39(3):924-929.

Wang Y., Ha U., Zeng L. \& Jin S. 2003. Regulation of membrane permeability by a two-component regulatory system in Pseudomonas aeruginosa. Antimicrob. Agents Chemother. 47:95-101. 